\title{
Relative Frequenzstabilitätsmessungen an Einmoden-Lasern
}

\author{
Bernd Wellegehausen und Arnold Güttner \\ Institut für Angewandte Physik der Technischen Universität Hannover
}

(Z. Naturforsch. 28 a, 968-972 [1973] ; eingegangen am 28. Februar 1973)

\begin{abstract}
The relative frequency stabilities and the modulation spectra of differently stabilized lasers are compared. The measurements were made by a Michelson interferometer with an internal optical delay line. For $6328 \AA \mathrm{He}-$ Ne-lasers stabilized by means of a Zeeman-absorption-cell and by the Lamb-dip-method frequency-standard-deviations of $1.3 \times 10^{5}$ and $9.0 \times 10^{5} \mathrm{~Hz}$ for observation times between $1 \mathrm{~s}$ and $20 \mathrm{~s}$ were measured. The frequency modulation spectra are composed of a low frequency noise part and many discrete frequencies, which can be related to the stabilization mechanism.
\end{abstract}

\section{Einleitung}

Die Frequenz eines Einmoden-Lasers ist abgesehen von Pulling- und Pushing-Effekten des Lasermediums im wesentlichen durch die optische Resonatorlänge bestimmt. Schwankungen der Resonatorlänge verursachen zeitliche Frequenzänderungen, die unter normalen Betriebsbedingugen des Lasers zu Linienbreiten führen, die groß sind im Vergleich zu den natürlichen Linienbreiten auf Grund des Quantenrauschens (spontane Emission) ${ }^{1}$. Der Einfluß von Amplitudenfluktuationen auf die Linienbreite kann im allgemeinen vernachlässigt werden. Ein Einmoden-Laser emittiert daher eine in der Amplitude praktisch konstante, in der Frequenz sich jedoch mehr oder weniger stark ändernde Lichtwelle.

Die zeitlichen Frequenzänderungen eines Lasers setzen sich zusammen aus Schwankungen um eine Zentralfrequenz infolge statistischer oder periodischer Störungen des Lasersystems (technisches Rauschen, z. B. mechanische Resonatorschwingungen, Brechungsindexfluktuationen) und einer langsamen Drift der Zentralfrequenz, hervorgerufen durch thermische Längenänderungen und Veränderungen im Lasermedium (z. B. Gasdruck und Gaszusammensetzung in Gaslasern) ${ }^{2,3}$. Insbesondere die thermischen Einflüsse führen dazu, daß die Frequenz eines freilaufenden Lasers in einem großen Bereich der Fluoreszenzlinie des Laserüberganges variiert. Beim $\mathrm{He}-\mathrm{Ne}$-Laser beträgt die Linienbreite des Dopplerverbreiterten $6328 \AA$-Laserüberganges z. B. $1,5 \cdot 10^{9}$ $\mathrm{Hz}$, bei Festkörperlasern weisen die Fluoreszenzlinienbreiten im allgemeinen um Größenordnungen höhere Werte auf. Mit Hilfe einer Frequenzstabilisie-

Sonderdruckanforderungen an Dr. B. Wellegehausen, Institut für Angewandte Physik der Technischen Universität Hannover, D-3000 Hannover, Welfengarten 1. rung ${ }^{2,3}$ gelingt es, den Variationsbereich der Laserzentralfrequenz innerhalb der Fluoreszenzlinie wesentlich einzuengen und damit die Frequenzgenauigkeit entsprechend zu erhöhen. Aussagen über die absolute Frequenzstabilität, d.h. Aussagen über langzeitige Veränderungen der Laserzentralfrequenz sind im optischen Bereich z. Zt. nur über den Vergleich mit dem Wellenlängenstandard möglich ${ }^{4,5}$. Hierbei ist jedoch auf Grund der Reproduzierbarkeit des Standards nur eine Auflösung von $10^{8}-10^{9} \mathrm{zu}$ erreichen ${ }^{2,3,4}$.

Relative Frequenzstabilitätsmessungen, wie z.B. Messungen der Standardabweichung der Frequenzfluktuationen in bezug auf die Zentralfrequenz, lassen sich dagegen mit einem sehr viel höheren Auflösungsvermögen durchführen. Derartige Messungen gestatten Aussagen über die Kohärenzlänge, die Linienbreite und die Linienform und damit über die Verwendungsmöglichkeiten des Lasers bei Präzisionslängenmessungen, als Frequenz-Referenzsystem oder in der hochauflösenden Absorptionsspektroskopie.

In diesem Artikel sollen Ergebnisse von relativen Frequenzstabilitäts- und Frequenzfluktuationsmessungen an zwei frequenzstabilisierten Einmoden-HeNe-Lasern (Typ LG 65: Fa. Siemens; Typ 119: Fa. Spectra Physics) mitgeteilt werden.

\section{Meßprinzip}

Hochauflösende relative Frequenzstabilitätsmessungen können mit Hilfe der Heterodyntechnik ${ }^{1}$ sowie mit interferometrischen Systemen durchgeführt werden ${ }^{6,7}$. Der Vorteil interferometrischer Meßmethoden liegt vor allem darin, daß ein passives Referenzsystem benutzt wird, das prinzipiell stabiler ausgeführt werden kann als ein Referenzlaser. 


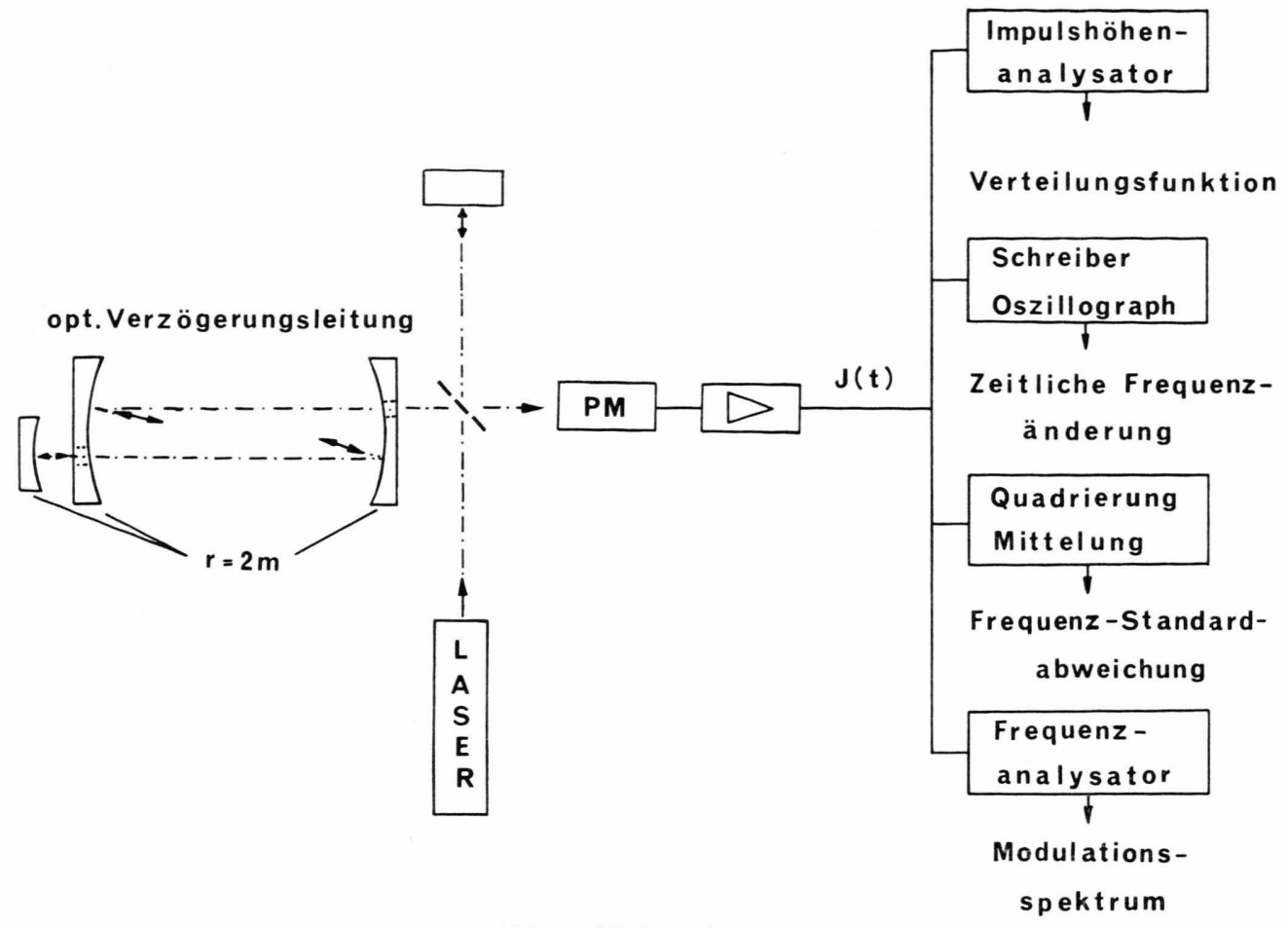

Für die hier beschriebenen Messungen wurde ein von Welling und Wellegehausen ${ }^{6}$ entwickeltes spezielles Michelson-Interferometer verwendet. Abb. 1 zeigt eine Prinzipdarstellung der Meßanordnung; detaillierte Angaben über das Meßsystem und seine Meßmöglichkeiten finden sich in der o. a. Arbeit. Das Michelson-Interferometer enthält eine optische Verzögerungsleitung ${ }^{8}$, die optische Wegdifferenzen von mehreren Kilometern ermöglicht. Über die Wegdifferenz kann dabei das Auflösungsvermögen dem jeweiligen Problem angepaßt werden.

Ändert sich die Frequenz des Lasers linear mit der Zeit, so liefert der Detektor in Abb. 1 wegen der gleichförmigen Verschiebung der Interferenzstreifen ein kosinusförmiges Signal $I(t)$ (siehe Abbildung 2). Der Übergang von einem Intensitätsminimum $\left(I_{\min }\right)$ zu einem benachbarten Intensitätsmaximum $\left(I_{\max }\right)$ entspricht jeweils einer Frequenzänderung $\Delta v$ mit dem Betrag $c / 2 \Delta s ; c$ ist dabei die Lichtgeschwindigkeit und $\Delta s$ die optische Wegdifferenz. Große monoton ablaufende Frequenzdrifts können daher einfach durch Zählen der auftretenden Intensitätsmaxima gemessen werden.

Im linearen Bereich der Intensitäts-FrequenzCharakteristik, in der Umgebung des Arbeitspunktes $I_{0}=\left(I_{\max }+I_{\min }\right) / 2$, sind die Intensitätsänderungen

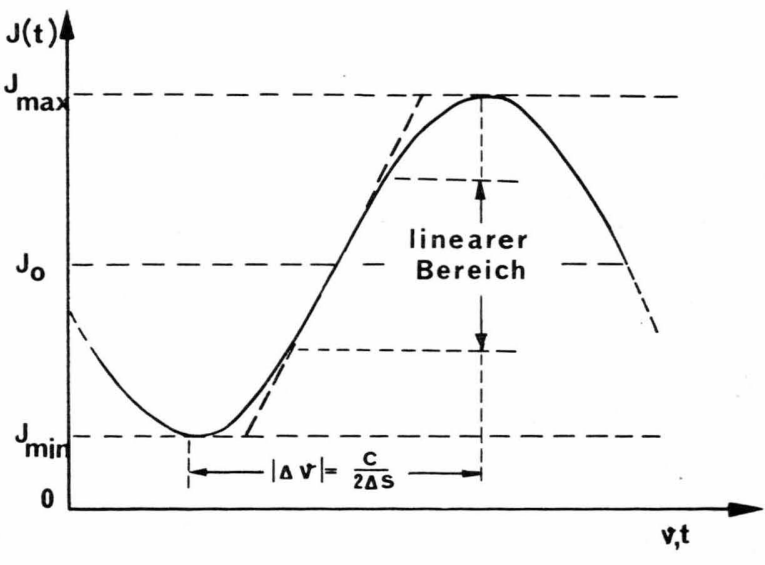

Abb. 2. Detektorsignal $I(t)$ bei gleichförmiger Frequenzdrift. In dem Bereich $\left(I_{0}+I_{\min }\right) / 2 \leqq I \leqq\left(I_{\max }+I_{0}\right) / 2$ (linearer Bereich) bleibt die Abweichung von der gestrichelt gezeichneten Geraden unter 5\%.

$\Delta I(t)$ den Frequenzschwankungen $v(t)$ proportional:

$$
\Delta I(t)=\pi I_{0} K(2 \Delta s / c) v(t) .
$$

$v^{\prime}(t)$ ist die Abweichung der momentanen Laserfrequenz von der Zentralfrequenz $v_{0}$ und $K$ steht für

$$
\left(I_{\max }-I_{\min }\right) /\left(I_{\max }+I_{\min }\right) \text {. }
$$

Eine Messung von $v(\mathrm{t})$ nach Formel 1 setzt voraus, daß die optische Wegdifferenz hinreichend kon- 
stant ist, d.h., daß die relativen Wegdifferenzfluktuationen klein sind gegenüber den nachzuweisenden relativen Frequenzänderungen. Durch einen besonders stabilen Interferometeraufbau konnte eine relative Weglängenstabilität von nahezu $10^{11}$ für Meßzeiten bis zu 10 s erreicht werden ${ }^{6}$. Eine Beschränkung der Meßzeit war auf Grund der thermischen Drift der optischen Weglänge notwendig.

Trotz der auf $10^{11}$ begrenzten Stabilität des Interferometers konnten mit einem leicht modifizierten System bei der Bestimmung der natürlichen Linienbreite eines $\mathrm{He}-\mathrm{Ne}$-Lasers Werte bis herab zu $250 \mathrm{~Hz}$ entsprechend einem Auflösungsvermögen von $2 \cdot 10^{12}$ gemessen werden ${ }^{9}$. Eine spezielle Meßmethode gestattete dabei eine weitgehende Reduzierung des Einflusses technischer Störungen im Interferometersystem und im untersuchten Laser.

\section{Meßergebnisse}

Für zwei auf verschiedene Weise frequenzstabilisierte $\mathrm{He}$ - Ne-Laser (Laser A: Lamb-dip-stabilisierter Laser Modell 119; Laser B: Zeeman-Absorptionszellen-stabilisierter Laser LG 65) ${ }^{2,3}$ wurden folgende das relative Frequenzverhalten charakterisierende Größen bestimmt:

1) Frequenz-Standardabweichung $\sigma_{v}=\sqrt{\left\langle v^{2}\right\rangle}$

$(\langle\cdots\rangle$ : Zeitmittelung) und relative Frequenzstabilität $v_{0} / \sigma_{v}$.

2) Spektrum der Frequenzfluktuationen $v(t)$. Dabei wird zwischen der Leistungsdichte $g_{v}(f)$ eines kontinuierlichen Rauschanteiles sowie den Frequenzhüben $\hat{v}\left(f_{i}\right)$ für diskrete Modulationsfrequenzen $f_{i}$ unterschieden.

3) Verteilungsfunktionen $W(v)$ der Frequenzabweichung $v(t)$.

Zur Bestimmung dieser Größen wurden jeweils geeignete optische Wegdifferenzen gewählt, die bei den verwendeten Lasern im Bereich von $5-50 \mathrm{~m}$ lagen.

In Tab. 1 sind die Standardabweichungen $\sigma_{v}$ und die relativen Stabilitäten $v_{0} / \sigma_{v}$ der Laser angegeben. Unter der Annahme einer gaußförmigen Laserlinie lassen sich aus $\sigma_{v}$ die ebenfalls in Tab. 1 aufgeführten Linienbreiten (Halbwertsbreiten) $\delta_{v}$ berechnen. Eine gaußsche Linienform ist stets zu erwarten, wenn die Frequenzfluktuationen des Lasers im wesentlichen durch voneinander unabhängige niederfrequente Störungen (technisches Rauschen) bewirkt werden ${ }^{1}$.

Detaillierte Aussagen über die Ursachen der Frequenzschwangungen lassen sich aus den Modula-
Tab. 1. Frequenz-Standardabweichungen, Linienbreiten und relative Stabilitäten für zwei Einmoden-He-Ne-Laser. Mittelungszeiten $1 \mathrm{~s}-20 \mathrm{~s} . v_{0}=4,74 \cdot 10^{14} \mathrm{~Hz}$.

\begin{tabular}{lllll}
\hline $\begin{array}{l}\text { Meß- } \\
\text { objekt }\end{array}$ & \multicolumn{2}{c}{$\begin{array}{c}\text { Frequenz-Standard- } \\
\text { abweichung } \sigma_{v} \\
\text { direkt } \\
\text { gemessen }\end{array}$} & $\begin{array}{l}\text { Lus Modu- } \\
\text { lations- } \\
\text { spektrum } \\
\text { beite } \\
\delta_{\nu}\end{array}$ & $\begin{array}{l}\text { relative } \\
\text { Stabilität } \\
v_{0} / \sigma_{\nu}\end{array}$ \\
\hline berechnet \\
Laser A \\
Laser B & $9,0 \cdot 10^{5} \mathrm{~Hz}$ & $1,0 \cdot 10^{6} \mathrm{~Hz}$ & $2,1 \cdot 10^{6} \mathrm{~Hz}$ & $5,3 \cdot 10^{8}$ \\
& $1,3 \cdot 10^{5} \mathrm{~Hz}$ & $1,4 \cdot 10^{5} \mathrm{~Hz}$ & $3,1 \cdot 10^{5} \mathrm{~Hz}$ & $3,6 \cdot 10^{9}$ \\
\hline
\end{tabular}

tionsspektren (Abb. 3, 4) ableiten. Die Spektren wurden mit einem Frequenzanalysator konstanter relativer Bandbreite aufgenommen und auf eine Bandbreite von $1 \mathrm{~Hz}$ reduziert. Durch eine Integration bzw. Summation ergeben sich aus den Spektren die Standardabweichungen $\sigma_{v} \mathrm{zu}$ :

$$
\sigma_{\nu}^{2}=\left\langle v^{2}\right\rangle=\int_{0}^{\infty} g_{v}(f) \mathrm{d} f+\frac{1}{2} \sum_{i} \hat{v}^{2}\left(f_{i}\right) .
$$

Die nach (2) berechneten Werte sind in Tab. 1 angegeben.

Die Abb. 5 und 6 zeigen Verteilungen $W(v)$ der Frequenzabweichung $v$. Die Registrierung erfolgte mit einem Vielkanalimpulshöhenanalysator. Die Verteilungen sind näherungsweise gaußförmig, was auf eine statistische Zusammensetzung der Frequenzkomponenten schließen läßt. Die Halbwertsbreiten stimmen gut mit den Linienbreiten der gaußschen Spektren (siehe Tab. 1) überein.

\section{Diskussion der Meßergebnisse}

Die gemessenen Werte für die Standardabweichungen der Frequenzfluktuationen bzw. die relativen Stabilitäten beziehen sich auf begrenzte Beobachtungszeiten. Das bedeutet, daß Frequenzkomponenten des Modulationsspektrums unterhalb einer Grenzfrequenz (hier z. B. unterhalb $10^{-2} \mathrm{~Hz}$ bei Meßzeiten bis zu 20 s) sowie eine thermische Frequenzdrift nicht bzw. nur teilweise erfaßt wurden. Sofern derartige Störungen nicht durch den Frequenzdiskriminator und den Regelungskreis selbst produziert werden, sollte sie jedoch die Regelung weitgehend eliminieren. Nach einer längeren Einlaufzeit des Lasers und bei konstanten Umgebungsbedingungen dürften die angegebenen Stabilitätswerte daher auch noch für wesentlich größere Beobachtungszeiträume gültig sein.

Die Hauptursachen der Frequenzfluktuationen lassen sich bei bekanntem Stabilisierungsmechanis- 

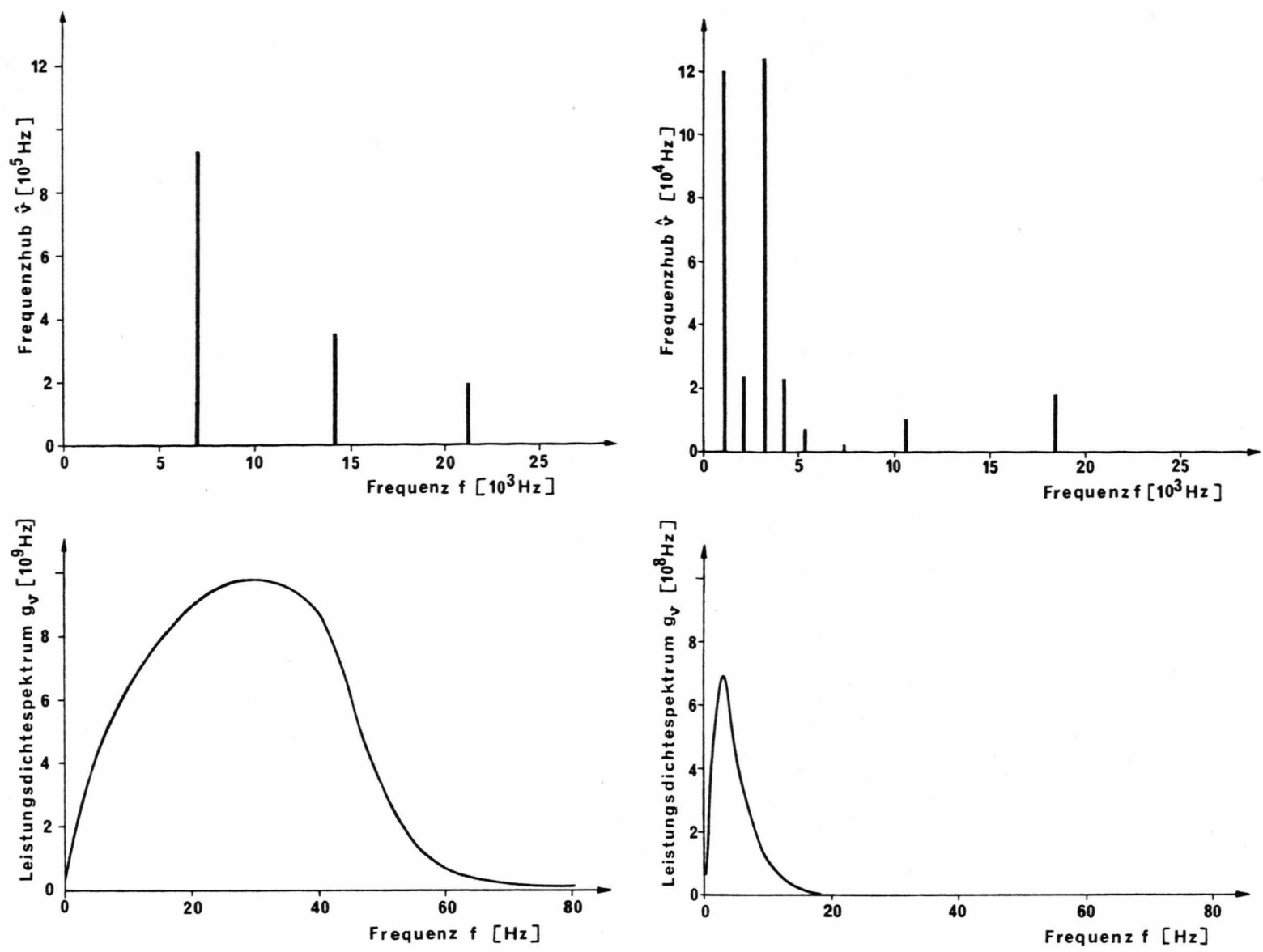

Abb. 3. Kontinuierliches und diskretes Modulationsspektrum des Lasers A (Lamb-dip-Stabilisierung).

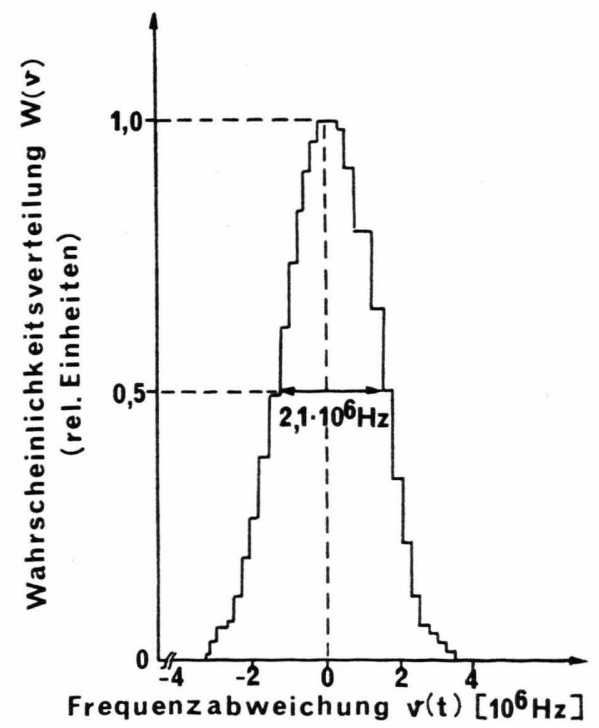

Abb. 5. Wahrscheinlichkeitsverteilung $W(v)$ der Frequenzabweichung $v(t)$ des Lasers A. Registrierdauer $60 \mathrm{~s}$.
Abb. 4. Kontinuierliches und diskretes Modulationsspektrum des Lasers B (Zeeman-Zellen-Stabilisierung). Ordinatenmaßstab gegenüber $\mathrm{Abb}$. 3 geändert.

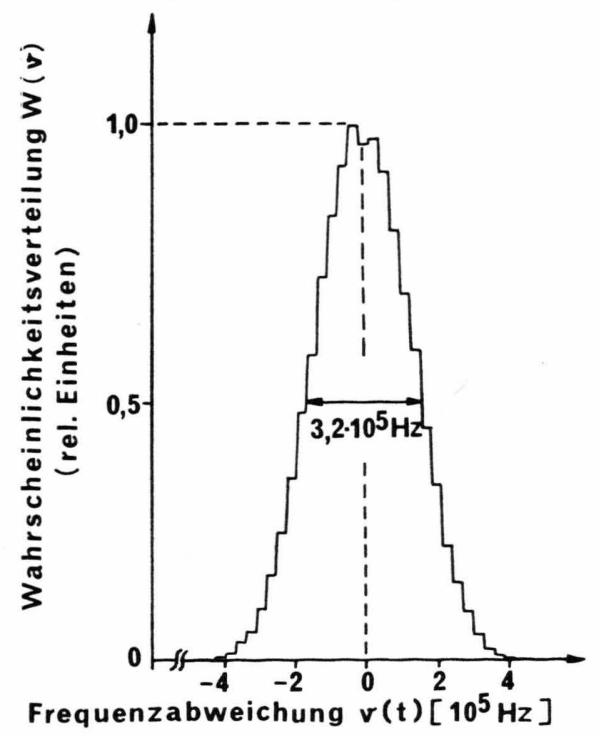

Abb. 6. Wahrscheinlichkeitsverteilung $W(v)$ der Frequenzabweichung $v(t)$ des Lasers B. Registrierdauer $10 \mathrm{~s}$. Abszissenmaßstab gegenüber $\mathrm{Abb} .5$ geändert. 
mus aus den Modulationsspektren entnehmen. Die Lamb-dip-Stabilisierung des Lasers A beruht darauf, daß die Laserfrequenz an der Stelle des lokalen Intensitästminimums (Lamb-dip) im frequenzabhängigen Intensitätsprofil festgehalten wird. Das Regelungssignal wird aus dem intensitätsmodulierten Signal gewonnen, das bei einer piezoelektrischen Wobbelung der Laserfrequenz entsteht $2,3,10$. Diese Wobbelung ist mit diskreten Frequenzen im Modulationsspektrum verbunden (Abb. 5). Der niederfrequente kontinuierliche Anteil des Modulationsspektrums dürfte dagegen vor allem auf statistische Resonatorinstabilitäten zurückgehen. Der Abfall dieses Spektrums zu niedrigen Frequenzen läßt die Wirkung der Regelung erkennen. Die diskreten Komponenten allein würden nach (2) eine Standardabweichung von $7 \cdot 10^{5} \mathrm{~Hz}$ ergeben. Die relative Stabilität dieses Lasers ist daher zu einem wesentlichen Teil durch die direkte Modulation der Laserfrequenz bestimmt, d.h. durch den Stabilisierungsmechanismus selbst. $\sigma_{v}$-Messungen am freilaufenden (unstabilisierten) Laser ergaben einen Wert von $7 \cdot 10^{4} \mathrm{~Hz}$ über eine Zeit von $10 \mathrm{~s}$.

Beim Laser B (Zeeman-Zellen-Stabilisierung) dient eine Absorptionszelle als Referenzsystem und als Frequenzdiskriminator 2, 3,11. Die Absorptionszelle befindet sich in einem magnetischen Wechselfeld, so daß auf Grund der Zeeman-Aufspaltung durch die Zelle hindurchtretendes zirkular polarisiertes Laserlicht intensitätsmoduliert wird. Aus der Intensitätsmodulation und der Phasenlage relativ zum Magnetfeld wird das Regelungssignal für die Frequenzstabilisierung abgeleitet ${ }^{11}$. Da hierbei keine Modulation der Laserfrequenz stattfindet, sollte sich eine bessere relative Stabilität als beim Laser A ergeben. Die in Tab. 1 aufgeführten Meßwerte bestätigen dies. Die kleineren $\sigma_{v}$-Werte sind sowohl auf die niedrigere Leistungsdichte des kontinuierlichen Modulationsspektrums (Regelungsbandbreite $<10 \mathrm{~Hz})^{11}$ als auch auf die erheblich kleineren Hübe der diskreten Modulationsfrequenzen zurückzuführen, die anders als bei der Lamb-dip-Sta-

1 A. E. Siegman, B. Daino u. K. R. Manes, IEEE J. Quantum Electron. 3, 180 [1967].

2 M. Gronchi u. A. Sona, Riv. Nuovo Cim. 2, 219 [1970].

${ }^{3}$ G. Birnbaum, Proc. IEEE 55, 1015 [1967].

4 E. Engelhard u. R. Vieweg, Z. Angew. Phys. 13, 580 [1961].

5 K. D. Mielenz, R. B. Stephens, K. E. Gilliland u. K. F. Nefflen, J. Opt. Soc. Amer. 56, 156 [1966].

6 H. Welling u. B. Wellegehausen, Appl. Opt. 11, 1986 [1972]. bilisierung nicht durch das Stabilisierungsprinzip bedingt sind. Abgesehen von einer Linie bei etwa $18,5 \mathrm{~Hz}$, die auch im unstabilisierten Zustand vorhanden ist und auf die Anregung der Gasentladung zurückgeht, sind alle übrigen Modulationsfrequenzen Harmonische der Magnetfeldfrequenz von $532 \mathrm{~Hz}$. Messungen ergaben einen nahezu linearen Zusammenhang zwischen der Magnetfeldstärke und den Frequenzhüben, die Auslegung des Stabilisierungskreises ließ jedoch keine wesentliche Erniedrigung der Magnetfeldstärke zu. Der Mechanismus, über den das Magnetfeld die Laserfrequenz beeinflußt, wurde nicht untersucht. Werden die mit dem Magnetfeld zusammenhängenden Störfrequenzen vernachlässigt, so liefert die Integration nach (2) eine Frequenz-Standardabweichung von $6 \cdot 10^{4} \mathrm{~Hz}$ bzw. eine relative Stabilität von $8 \cdot 10^{9}$. Stabilitätsmessungen am freilaufenden Laser ergaben einen Wert von $3 \cdot 10^{10}$ über $10 \mathrm{~s}$.

\section{Zusammenfassung}

Mit Hilfe eines Michelson-Interferometers sind bei geeigneter Wahl der optischen Wegdifferenz detaillierte Aussagen über das zeitliche Frequenzverhalten von Lasern möglich. Für zwei nach verschiedenen Methoden frequenzstabilisierte $\mathrm{He}-\mathrm{Ne}$-Laser wurden relative Stabilitäten (Linienbreiten) sowie die Modulationsspektren der zeitlichen Frequenzschwankungen der Laser bestimmt. Die Kenntnis dieser Spektren ist dabei von besonderer Bedeutung für das Auffinden und die Beseitigung von Störeinflüssen. Die Messungen ergaben, daß die relative Frequenzstabilität der untersuchten Laser hauptsächlich durch diskrete Störfrequenzen, die von den Stabilisierungsmechanismen selbst erzeugt werden, begrenzt ist. Damit besteht prinzipiell die Möglichkeit, die Frequenzstabilität dieser Laser weiter $\mathrm{zu}$ verbessern.

Die untersuchten Laser wurden uns freundlicherweise von der Physikalisch-Technischen Bundesanstalt und der Fa. Siemens zur Verfügung gestellt.

7 R. L. Fork, D. R. Herriott u. H. Kogelnik, Appl. Opt. 3, 1471 [1964].

8 D. R. Herriott u. H. J. Schulte, Appl. Opt. 4, 883 [1965].

${ }^{9}$ H. Gerhardt, H. Welling u. A. Güttner, Z. Phys. 253, 113 [1972].

10 Spectra Physics, Bedienungsanleitung.

11 V. Bodlaj, Frequenz 23, 92 [1969]; Frequenz 23, 374 [1969]; Dissertation TH München 1970. 